
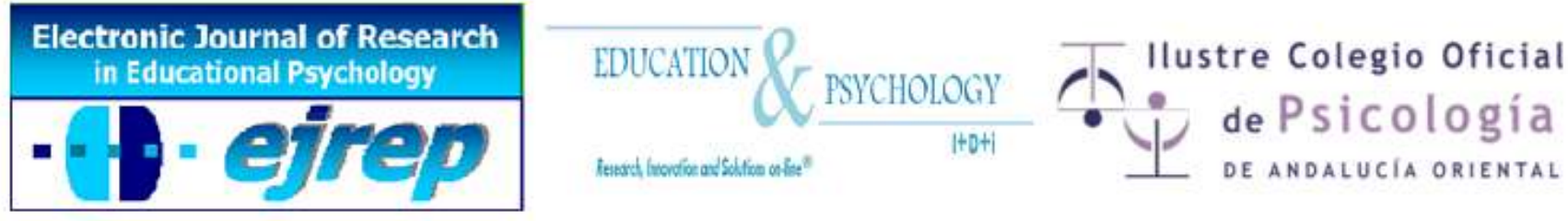

\title{
Presentación del Monográfico Emociones académicas en la Universidad
}

\author{
Jesús de la Fuente Arias \\ (Coordinador)
}

Departamento de Psicología. Universidad de Almería.

\section{España}




\section{Presentación}

La investigación sobre las emociones académicas se ha convertido en un tópico de investigación relevante en los últimos años (Davis y Levine, 2013; Romero et al., 2014), alentada por la evolución de los paradigmas de procesamiento de la información -casi exclusivamente centrados en variables cognitivas y metacognitivas- hacia otros de última generación que contemplan la relevancia de las variables emocionales y meta-emocionales. Así, se están incorporando diversas variables emocionales (auto-regulación personal, confianza académica, estrategias de afrontamiento, resiliencia o engagement) a los modelos cognitivos y metacognitivos clásicos, evaluando su relevancia e interacción con las variables cognitivas y el rendimiento académico (de la Fuente et al., 2015; Bembenutty, Cleary y Kintasas, 2013; Chisteson, Reschly y Wylie, 2013). También, en el ámbito universitario, se está constatando la importancia de la investigación y del conocimiento sobre el papel de estas variables emocionales en procesos cognitivos y metacognitivos (Artino et al., 2014; Pekrun, 2005; Pekrun et al., 2007; Villavicencio y Bernardo, 2013).

El presente monográfico, que hemos denominado Emociones Académicas en la Universidad - sin ánimo de ser exhaustivo-, pretende contribuir a la revisión del estado de este conocimiento, así como divulgar las nuevas evidencias de la investigación y del desarrollo de la innovación tecnológica reciente, en este tópico de investigación. Aborda diferentes aspectos. En la primera parte, de revisión del tópico, se sitúa el primer artículo en el que se realiza un análisis de la importancia y evolución de este tópico de investigación. Una segunda parte aporta trabajos de evidencia empírica. El segundo artículo, muestra el efecto interactivo de la autorregulación personal, como variable del alumnado, y de la enseñanza reguladora, como variable del contexto, para establecer el efecto en la confianza académica de los estudiantes, tras un proceso de enseñanza-aprendizaje universitario. El tercer artículo, aporta evidencia de las relaciones entre la resiliencia y las estrategias de afrontamiento, mostrando los diferentes perfiles de la misma, según el contexto formativo, laico o religioso de los estudiantes universitarios, a la hora de hacer frente al estrés académico. Un cuarto artículo, muestra evidencia de las relaciones entre burnout y adquisición de las competencias en la Universidad. La tercera parte, en sendos artículos, presenta trabajos de validación de dos instrumentos que permiten evaluar las emociones académicas en la universidad. Finalmente, 
la última sección está dedicada a la presentación de una nueva e-herramienta de autoayuda para evaluar y mejorar los comportamientos de afrontamiento del estrés académico universitario, en situaciones de estudio u oposición.

\section{Agradecimientos}

Este número Monográfico se ha desarrollado gracias al Proyecto I+D ref. EDU201124805 (2012-2014). MICINN (España) y Fondo Social Europeo (UE).

\section{Referencias}

Artino, A.IR., La Rochelle, J.S. y Durning, S.J. (2010). Second-year medical students' motivational beliefs, emotions, and achievement. Med Educ 44(12):1203-1212.

Bembenutty, H., Cleary, T. y Kintasas, A. (2013). Applications of Self-Regulated Learning across Diverse Disciplines. A tribute to Barry Zimmerman. Charlotte, NY: Information Age Publishing, Inc.

Chase P.A., Hilliard, L.J., John Geldhof, G., Warren, D.J. y Lerner, R.M. (2014). Academic achievement in the high school years: the changing role of school engagement. $J$ Youth Adolesc, 43(6), 884-896.

Chisteson, S. L., Reschly, A.L. y Wylie, C. (2013). Handbook of Research on Student Engagement. London: Springer.

De la Fuente, J., Martínez-Vicente, J.M., Zapata, L., Sander, P. y Putwain, D. (2015). Personal Selfregulation, Self-regulated Learning and Coping Strategies, in University Context with Stress. In A. Peña-Ayala (Ed.), Metacognition: Fundaments, Applications, and Trends. A Profile of the Current State-Of-The-Art. NY: Springer.

Davis, E.L. y Levine, L.J. (2013). Emotion regulation strategies that promote learning: reappraisal enhances children's memory for educational information. Child Dev, 84(1), 361-374.

Pekrun, R. (2005). Progress and open problems in educational emotion research. Learning and Instruction, 15, 497-506. 
Pekrun, R., Goetz, T., Frenzel, A., Barchfeld, P. y Perry, R. (2011). Measuring emotions in students` learning and performance: The Achievement Emotions Questionnaire (AEQ). Contemporary Educational Psychology, 36, 36-48.

Romero C., Master A., Paunesku D., et al. (2014). Academic and emotional functioning in middle school: The role of implicit theories. Emotion, 14(2), 227-234.

Villavicencio, FT. y Bernardo, A.B. (2013). Positive academic emotions moderate the relationship between self-regulation and academic achievement. British Journal of Educational Psychology, 83(2), 329-340. 